\title{
Soft X-ray emission lines in the X-ray binary Swift J1858.6-0814 observed with XMM-Newton Reflection Grating Spectrometer: disc atmosphere or wind?
}

\author{
D. J. K. Buisson, ${ }^{1 \star}$ D. Altamirano, ${ }^{1}$ M. Díaz Trigo,${ }^{2}$ M. Mendez, ${ }^{3}$ M. Armas Padilla ${ }^{\circledR}, 4$ \\ N. Castro Segura ${ }^{\circledR},{ }_{1}^{1}$ N. D. Degenaar, ${ }^{6}$ J. van den Eijnden ${ }^{\circledR},{ }^{6}$ F. A. Fogantini, ${ }^{7,8}$ P. Gandhi ${ }^{\circledR}, 1$ C. Knigge, ${ }^{1}$ \\ T. Muñoz-Darias ${ }^{\circledR}, 4,5$ M. Özbey Arabac1 ${ }^{1,9}$ and F. M. Vincentelli ${ }^{\circledR 1}$ \\ ${ }^{1}$ Department of Physics and Astronomy, University of Southampton, Highfield, Southampton SO17 1BJ, UK \\ ${ }^{2}$ ESO, Karl-Schwarzschild-Strasse 2, D-85748 Garching bei München, Germany \\ ${ }^{3}$ Kapteyn Astronomical Institute, University of Groningen, PO Box 800, NL-9700 AV Groningen, the Netherlands \\ ${ }^{4}$ Instituto de Astrofísica de Canarias, Universidad de La Laguna, E-38205 La Laguna, Tenerife, Spain \\ ${ }^{5}$ Departamento de Astrofísica, Universidad de La Laguna, E-38206 La Laguna, Tenerife, Spain \\ ${ }^{6}$ Anton Pannekoek Institute for Astronomy, University of Amsterdam, Science Park 904, NL-1098 XH, Amsterdam, the Netherlands \\ ${ }^{7}$ Facultad de Ciencias Astronómicas y Geofísicas, Universidad Nacional de La Plata, Paseo del Bosque s/n, 1900 La Plata, Argentina \\ ${ }^{8}$ Instituto Argentino de Radioastronomía (CCT-La Plata, CONICET; CICPBA), C.C. No. 5, 1894 Villa Elisa, Argentina \\ ${ }^{9}$ Department of Astronomy and Astrophysics, Atatürk University, Erzurum 25240, Turkey
}

Accepted 2020 July 25. Received 2020 July 13; in original form 2020 February 18

\begin{abstract}
We find soft X-ray emission lines from the X-ray binary Swift J1858.6-0814 in data from XMM-Newton Reflection Grating Spectrometer (RGS): N VII, O VII, and O VIII, as well as notable residuals short of a detection at Ne IX and other higher ionization transitions. These could be associated with the disc atmosphere, as in accretion disc corona sources, or with a wind, as has been detected in Swift J1858.6-0814 in emission lines at optical wavelengths. Indeed, the N VII line is redshifted, consistent with being the emitting component of a P-Cygni profile. We find that the emitting plasma has an ionization parameter $\log (\xi)=$ $1.35 \pm 0.2$ and a density $n>1.5 \times 10^{11} \mathrm{~cm}^{-3}$. From this, we infer that the emitting plasma must be within $10^{13} \mathrm{~cm}$ of the ionizing source, $\sim 5 \times 10^{7} \mathrm{r}_{\mathrm{g}}$ for a $1.4 \mathrm{M}_{\odot}$ neutron star, and from the line width that it is at least $10^{4} \mathrm{r}_{\mathrm{g}}$ away $\left[2 \times 10^{9}\left(M / 1.4 \mathrm{M}_{\odot}\right) \mathrm{cm}\right]$. We compare this with known classes of emission-line regions in other X-ray binaries and active galactic nuclei.
\end{abstract}

Key words: accretion, accretion discs - black hole physics - stars: neutron-X-rays: binaries.

\section{INTRODUCTION}

A variety of emission and absorption lines are frequently observed in X-ray binaries; these provide constraints on various aspects of the system. Several physical components have the necessary conditions to produce these lines: particularly the plasma above the accretion disc. This may be the optically thin upper layers of the accretion flow, as seen in X-ray emission lines from accretion disc corona (ADC) sources (e.g. Cottam et al. 2001; Kallman et al. 2003), in which the surface layers of the disc evaporate and become extended (White \& Holt 1982; Miller \& Stone 2000; Jimenez-Garate, Raymond \& Liedahl 2002). Alternatively, it may be from a disc wind moving away from the central source (e.g. Ueda et al. 1998). Such winds are an important part of many accreting systems: Their power and mass flow rates can be comparable to the luminous power and mass accretion rate (Neilsen, Remillard \& Lee 2011; Ponti et al. 2012; Casares et al. 2019) and they can have a significant impact on their environment (e.g. Fender \& Muñoz-Darias 2016). However, many aspects of the origin and geometry of winds are still uncertain. Differentiating when

${ }^{\star}$ E-mail: d.j.k.buisson@soton.ac.uk material is ejected in a wind and when it remains bound to the system can show the conditions under which winds occur and hence help to determine how they are driven.

There are several means of driving X-ray binary winds: magnetocentrifugal acceleration of gas (e.g. Blandford \& Payne 1982; Miller et al. 2006a); radiation pressure (e.g. Icke 1980; Proga \& Kallman 2002); and thermal expansion of the atmosphere (e.g. Begelman, McKee \& Shields 1983; Woods et al. 1996). To distinguish between these requires observational constraints on plasma properties which differ between the possibilities.

In the X-ray band, winds are most commonly inferred from blueshifted absorption features (e.g. Díaz Trigo \& Boirin 2013); these include highly ionized species (e.g. Fe XXV/Fe XXVI). Deep observations show that X-ray absorbing winds can consist of several distinct components, with higher ionization typically occurring at higher velocity (e.g. Miller et al. 2016). Sometimes these features also have evidence for corresponding redshifted emission (e.g. Schulz \& Brandt 2002; King et al. 2015; Miller et al. 2015), giving the full line a P-Cygni profile.

Winds are often also detected as asymmetric emission features from recombination lines in the optical, which may have absorption in their blue wings, (Muñoz-Darias et al. 2016; Muñoz-Darias, 
Torres \& Garcia 2018), again forming a P-Cygni profile. The red emission/blue absorption 'P-Cygni' profile is expected from outflows such as disc winds (e.g. Dorodnitsyn 2009, 2010; Puebla et al. 2011) as only material in front of the source, moving towards the observer, absorbs the primary continuum while material moving away from the observer re-emits light in all directions.

Whether the winds detected in the optical and X-ray wavebands are manifestations of the same process or separate phenomena, possibly with different launching mechanisms, is not yet known. Observing wind properties of the same object in multiple wavebands is key to resolving this.

\subsection{Swift J1858.6-0814}

Swift J1858.6-0814 is a recently discovered (Krimm et al. 2018) $\mathrm{X}$-ray binary which shows P-Cygni line profiles in its optical spectra (Munoz-Darias et al. 2019; Muñoz-Darias et al. 2020). Its outburst has been long-lasting, being first detected on 2018 October 25 (Krimm et al. 2018) and ongoing at the time of writing (Hare et al. 2019; Rajwade et al. 2019; Buisson et al. 2020a), which has provided the opportunity for extensive observations with many instruments across a wide range of bandpasses. Recent detections of Type I Xray bursts show that Swift J1858.6-0814 has a neutron star accretor (Buisson et al., submitted); these bursts exhibit photospheric radius expansion, allowing a distance estimate of $\sim 15 \mathrm{kpc}$; and periodic eclipses have been found, showing that Swift J1858.6-0814 is viewed at high inclination (Buisson et al. 2020b). Before 2020, a common property of essentially all observations was atypically large variability.

Radio emission showed variability by up to a factor of 7-8 within 20 min and is consistent with a compact jet; the variability is likely due to mass accretion rate fluctuations rather than discrete ejecta (Bright et al. 2018; van den Eijnden et al. 2020). Optical variability included changes by factors of several within minutes (Vasilopoulos, Bailyn \& Milburn 2018) and fast flares, which are stronger at longer wavelengths (Paice et al. 2018). The wind absorption also changed in strength and terminal velocity, with changes not clearly related to the continuum variability (Muñoz-Darias et al. 2020). Such changes also occur in other systems, (e.g. Prinja et al. 2000; Kafka \& Honeycutt 2004).

The rapid X-ray variability in Swift J1858.6-0814 was extremely strong, occasionally changing by a factor of more than 200 within a few seconds (Fogantini et al., in preparation), which contrasted with the relatively stable long-term average flux. The X-ray spectra of Swift J1858.6-0814 are also unusual in their hardness $(\Gamma \sim 1.5)$ and show strong, variable obscuration (Reynolds et al. 2018; Hare et al. 2020). This is reminiscent of previous outbursts of the unusual X-ray binaries V4641 Sgr (Wijnands \& van der Klis 2000; Revnivtsev et al. 2002) and V404 Cyg (Gandhi et al. 2016; Motta et al. 2017; Walton et al. 2017)

In this paper, we present high-resolution soft X-ray spectra of Swift J1858.6-0814 from the XMM-Newton Reflection Grating Spectrometer (RGS). We describe the available observations and their reduction in Section 2, give results in Section 3, and consider their implications in Section 4.

\section{OBSERVATIONS AND DATA REDUCTION}

We analyse the two XMM-Newton (Jansen et al. 2001) observations of Swift J1858.6-0814, focussing on the RGS data. These have ObsIDs 0831791201 and 0831791701, taken on 2018 March 23 and April 13, respectively. Hereafter, we refer to these as observations
201 and 701. We reduced the RGS data with the XMM-Newton SAS, version 17.0.0. Using RGSPROC, we extracted source spectra from regions including 95 per cent of the Point Spread Function (PSF) and the background from outside of 99 per cent. We do this for both RGS 1 and 2 and spectral orders 1 and 2. This produced spectra with exposures of 58 and $45 \mathrm{ks}$, respectively.

To retain the maximum information in the spectrum, we use the unbinned data, discarding channels labelled by RGSPROC as bad. When fitting, we leave the different cameras, spectral orders, and observations as separate data sets, fitting jointly where relevant. The low counts in each channel necessitates using the statistic of Cash (1979), which treats the Poisson data accurately. We perform fits in ISIS version 1.6.2-43 (Houck \& Denicola 2000).

We also extract a $0.2-10 \mathrm{keV}$ light curve from the pn data for illustrative purposes, using EVSELECT and EPICLCCORR. We include events with PATTERN $<=4$, using columns 33-41 for the source and 55-63 as background (the observation was taken in timing mode).

\section{RESULTS}

We begin by showing the light curve of each observation (Fig. 1). This shows strong, rapid flaring as occurs throughout the outburst of Swift J1858.6-0814. This flaring dominates the variability while the long-term flux is more stable. Therefore, we initially produce a mean spectrum for the whole campaign (the effect of variability on the spectrum is considered in Section 3.2). The most prominent features in the RGS spectrum are several apparent emission lines (Fig. 2); the significance of these lines and the conclusions which can be inferred from them are the focus of this paper. To give a visual overview of the spectral shape, we also show the mean RGS spectrum of both observations combined, summed across both detectors and orders, as raw counts and unfolded to a constant model (in $v F v$, i.e. a $\Gamma=$ 2 power law), in the upper and middle panels of Fig. 3.

\subsection{Identification of emission lines}

To consider the significance of the emission lines in an unbiased way, we begin by performing a line scan, adding an unresolved Gaussian of fixed wavelength to a smooth continuum and recording the fit improvement at each wavelength. We use a continuum of a blackbody, since this was found to be the dominant spectral component at these energies by Hare et al. (2020). We also tried including a power law (which is dominant at higher energies) or using an absorbed disc-blackbody, but this turns out to make negligible difference.

The resulting fit improvements are shown in the bottom panel of Fig. 3. This shows clearly detected lines at around the rest wavelengths of O VII and N VII. Since these are known wavelengths of two of the most common lines, one need not consider the lookelsewhere effect for the full wavelength range tested. There is also a weaker feature at $\mathrm{O}$ VIII; the fit improvement would correspond to approximately $3 \sigma$ if treating the change in statistic as a simple likelihood ratio, but since this is not always reliable (e.g. Protassov et al. 2002), more careful treatment of the significance is required. Furthermore, there are many features with a change of fit statistic, $\Delta C$, between 4 and 9 . These are not robust detections as the number of wavelengths tested (several hundred resolution elements) would be expected to produce several such features by chance. However, they will still play a role in determining the best-fitting plasma models, so we note the ion of the common transition which fits each such feature most closely in Fig. 3 . 


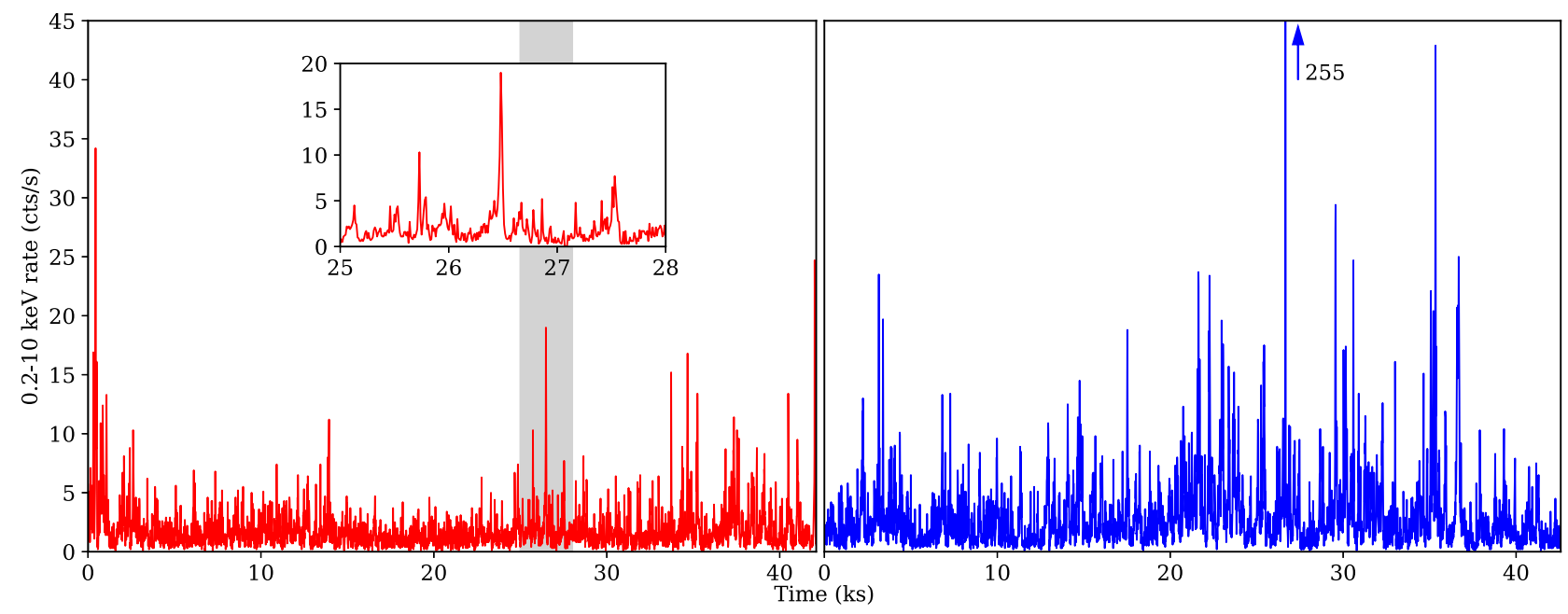

Figure 1. XMM-Newton-pn light curve of Swift J1858.6-0814. Observations 0831791201 and 0831791701 are shown in red and blue, respectively; they are separated by around $20 \mathrm{~d}$. The inset shows a zoom of the region marked in grey. The flare marked with the arrow reaches 255 counts s ${ }^{-1}$. The frequent, rapid flaring displayed in these observations is typical of Swift J1858.6-0814.

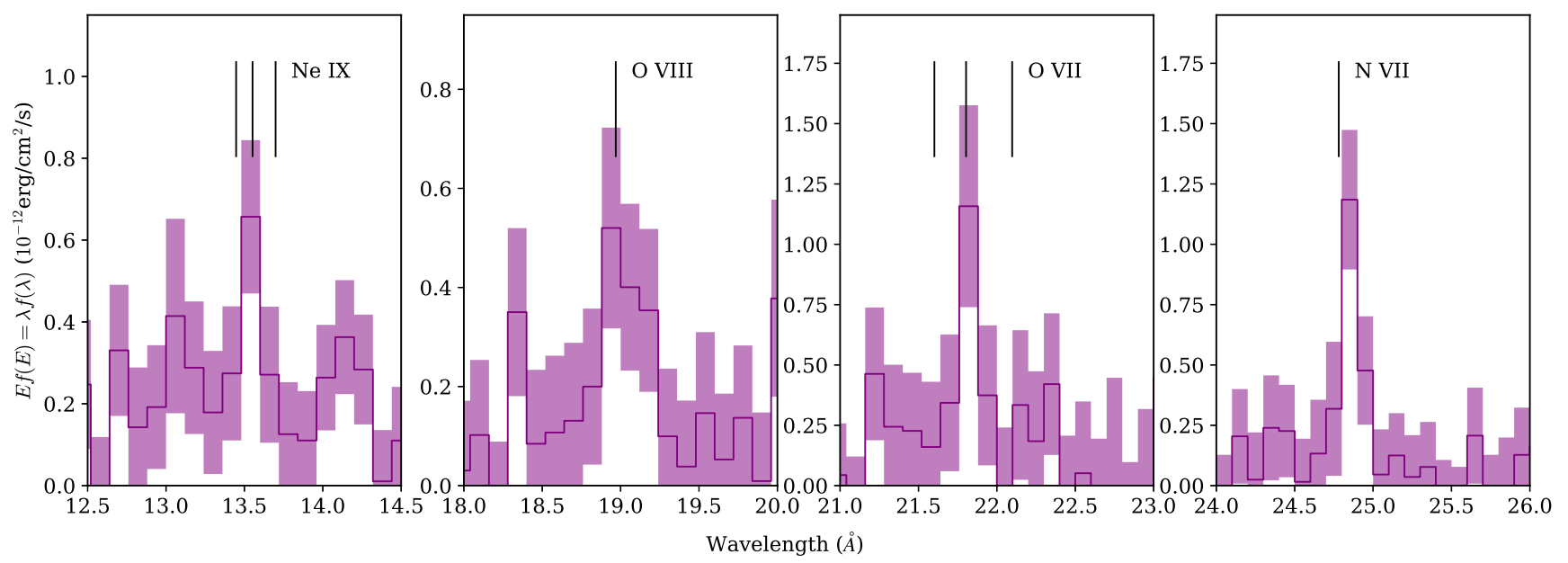

Figure 2. Continuum subtracted plots of the mean spectrum of both observations unfolded to a smooth model. Each panel shows a region of the spectrum containing a likely line; the most probable transition, along with its rest wavelength, is indicated.

\subsection{Significance and properties of lines}

We now perform a more detailed analysis of the most likely lines. We consider the clearly detected lines $(\Delta C>9)-\mathrm{O}$ VIII, O VII, and N VII - along with the strongest tentative line, Ne IX (apart from Si XIV, which is at the edge of the bandpass).

For each line, we use local models of a smooth continuum along with a narrow line. We fit a power law to the data within the region covering $\pm 2 \AA$ of the line, with a Gaussian allowed to be broadened and have a small redshift from the rest wavelength of the line. For the He-like ions (Ne IX and O VII), we use a triplet of three Gaussians with a common width and redshift, but independent strengths. We generate posterior distributions for line parameters with Markov chain Monte Carlo (MCMC) chains. For each line, we produce an MCMC chain for each observation separately and for both observations combined. We generate chains with 100 walkers per free parameter and 5000 steps, using the implementation in ISIS of the implementation of Foreman-Mackey et al. (2013) of the method of Goodman \& Weare (2010). We use flat (uninformative) priors on each parameter. We allow the line strength to be positive or negative such that the resulting posterior distribution gives the probability of an emission feature. The resulting parameters are shown in Table 1 and Fig. 4; we do not show $\mathrm{Ne}$ IX in the table since the inferred line strength includes 0 , so the width and redshift are not meaningful. We find that the probabilities of each line showing emission match the order of the $\Delta C$ values: Ne IX has only a 75 percent chance of being an emission line; the $\mathrm{N}$ VII line is the most likely at 99.97 per cent.

We also extract posteriors on the width and redshift of the lines. We find that in most cases, the values are consistent with being at rest However, the $\mathrm{N}$ VII line shows evidence for being redshifted $[P(z>$ $0)=0.94]$ and if the lines are assumed to have the same redshift, the combined probability reinforces this to $P(z>0)=0.96$. This may have a contribution from instrumental systematic errors or the source radial velocity, but if intrinsic to the source could be because the full line profile is a P-Cygni profile from a wind, with the absorbed blue side of the line shifting the line centroid redward.

We also test for variability of the detected lines by comparing the posteriors between the two observations. For each species, the 90 per cent credible intervals from the two observations overlap, so the line strengths are consistent with remaining constant between the 


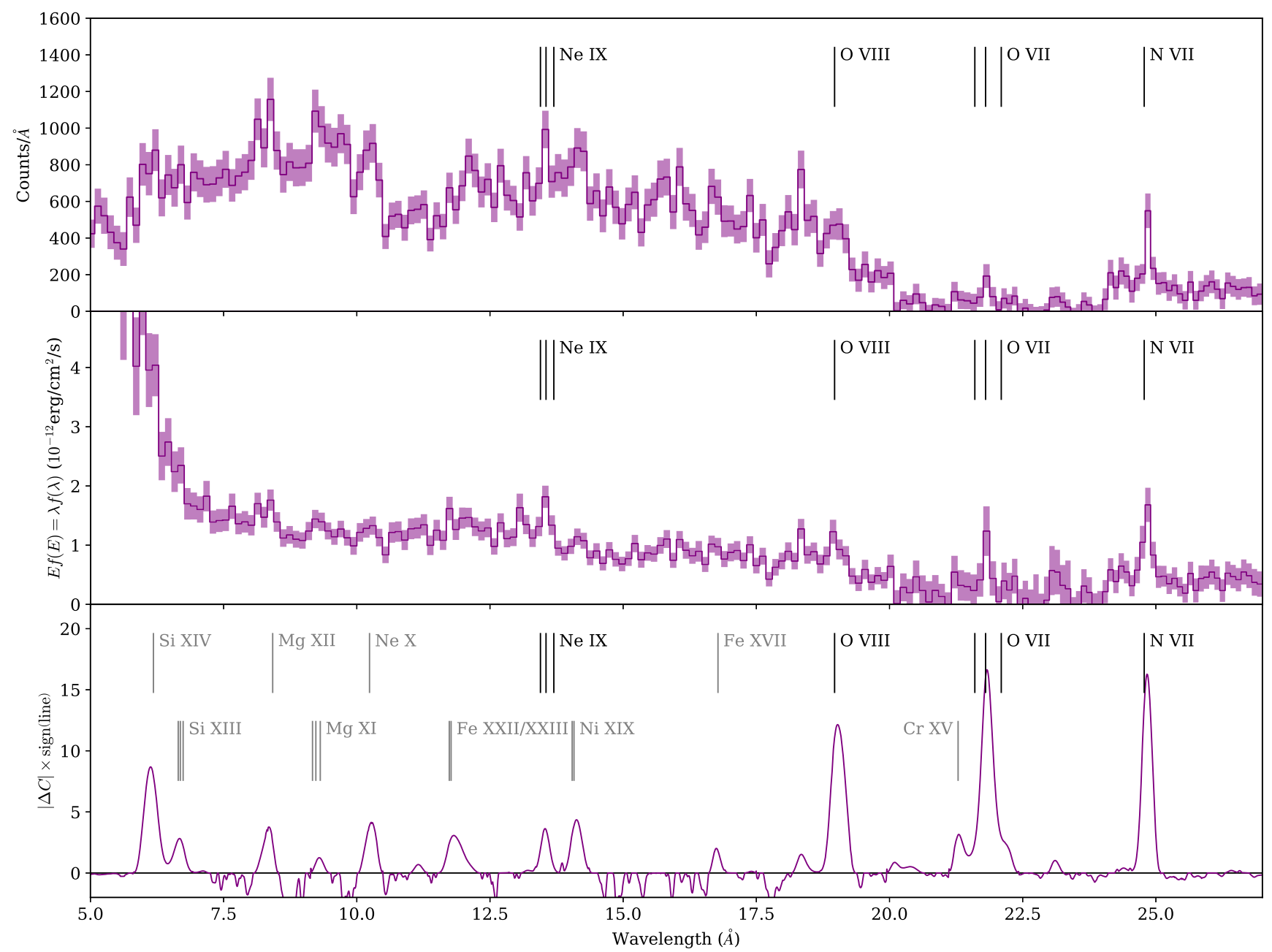

Figure 3. Combined RGS spectrum of Swift J1858.6-0814 (rebinned for plotting). Top row: Background subtracted spectrum of both observations combined, shown as detected counts summed over all detectors and orders. Second row: unfolded to a $\Gamma=2$ power law to remove the effect of the detector effective area. Bottom row: improvement in fit statistic from adding a narrow Gaussian to a continuum model. The magnitude gives $\Delta C$ and the sign shows the nature of the additional line: positive represents additional emission; negative represents absorption. O VII and N VII are clearly detected; given these, O VIII is detected at the expected wavelength. Plausible transitions (grey) exist for all features with $\Delta C>4$.

Table 1. Properties of emission lines in the combined RGS data. Errors are 90 per cent credible intervals.

\begin{tabular}{lccc}
\hline $\begin{array}{l}\text { Ion } \\
\text { Transition }\end{array}$ & $\begin{array}{c}\text { O VIII } \\
1 \mathrm{~s}^{1}-2 \mathrm{p}^{1}\end{array}$ & $\begin{array}{c}\mathrm{O} \text { VII } \\
1 \mathrm{~s}^{2}-1 \mathrm{~s}^{1} 2 \mathrm{p}^{1}\end{array}$ & $\begin{array}{c}\mathrm{N} \text { VII } \\
1 \mathrm{~s}^{1}-2 \mathrm{p}^{1}\end{array}$ \\
\hline$P($ Line strength $>0)$ & 0.989 & 0.994 & 0.9997 \\
Line strength $\left(10^{-6}\right.$ photon $\left.\mathrm{cm}^{-2} \mathrm{~s}^{-1}\right)$ & $8_{-7}^{+10}$ & $13_{-8}^{+9}$ & $9_{-4}^{+5}$ \\
Redshift $\left(10^{-3}\right)$ & $1 \pm 9$ & $1.5_{-4.2}^{+5.6}$ & $2.2_{-2.0}^{+2.2}$ \\
Velocity $\left(\mathrm{km} \mathrm{s}^{-1}\right)$ & $400_{-2700}^{+2900}$ & $450_{-1250}^{+1700}$ & $650_{-600}^{+680}$ \\
Width $(\AA)$ & $<0.27$ & $<0.11$ & $<0.13$ \\
Width $\left(\mathrm{km} \mathrm{s}^{-1}\right)$ & $<4300$ & $<1600$ & $<1600$ \\
\hline
\end{tabular}

observations. Similarly for no line, there is a significant probability that one particular observation is brighter than the other (the probabilities that observation 201 has a brighter line than observation 701 being: 0.43 for Ne IX, 0.08 for O VIII, 0.17 for O VII, and 0.86 for $\mathrm{N}$ VII). We also search for variability of the lines within an observation, in particular, with the continuum flux. We do this by splitting the RGS exposure based on the $0.7-10 \mathrm{keV}$ flux from the pn data. Even when split into only two flux states, this does not place interesting constraints on the variability in terms of either line flux or equivalent width.

\subsection{Gas density from the O VII triplet}

To derive physical properties of the plasma, we can either consider the most important transitions individually or use models of the full spectrum. Full models maximize the spectral information used and reduce the effect of possible contamination of an individual feature but require more assumptions (for example relative abundances) about the plasma. We begin by considering parameters which may be constrained using the most strongly detected transitions and model the full spectrum in Section 3.5.

The O VII feature at around $22 \AA$ is actually a triplet, with components at $21.60,21.80$, and $22.10 \AA$. The relative strength of these components is sensitive to the ionization mechanism and density of the gas (Porquet et al. 2001). The resonance ( $r, w$, $22.1 \AA$ ) line is strong in collisional-dominated (thermally ionized) plasmas and weak in photoionized plasmas. The relative strength of the forbidden $(f, z, 21.6 \AA)$ and intercombination $(i, x+y, 21.8 \AA)$ lines depends on the density, with forbidden being strong at low density and intercombination and high density (the relevant density 


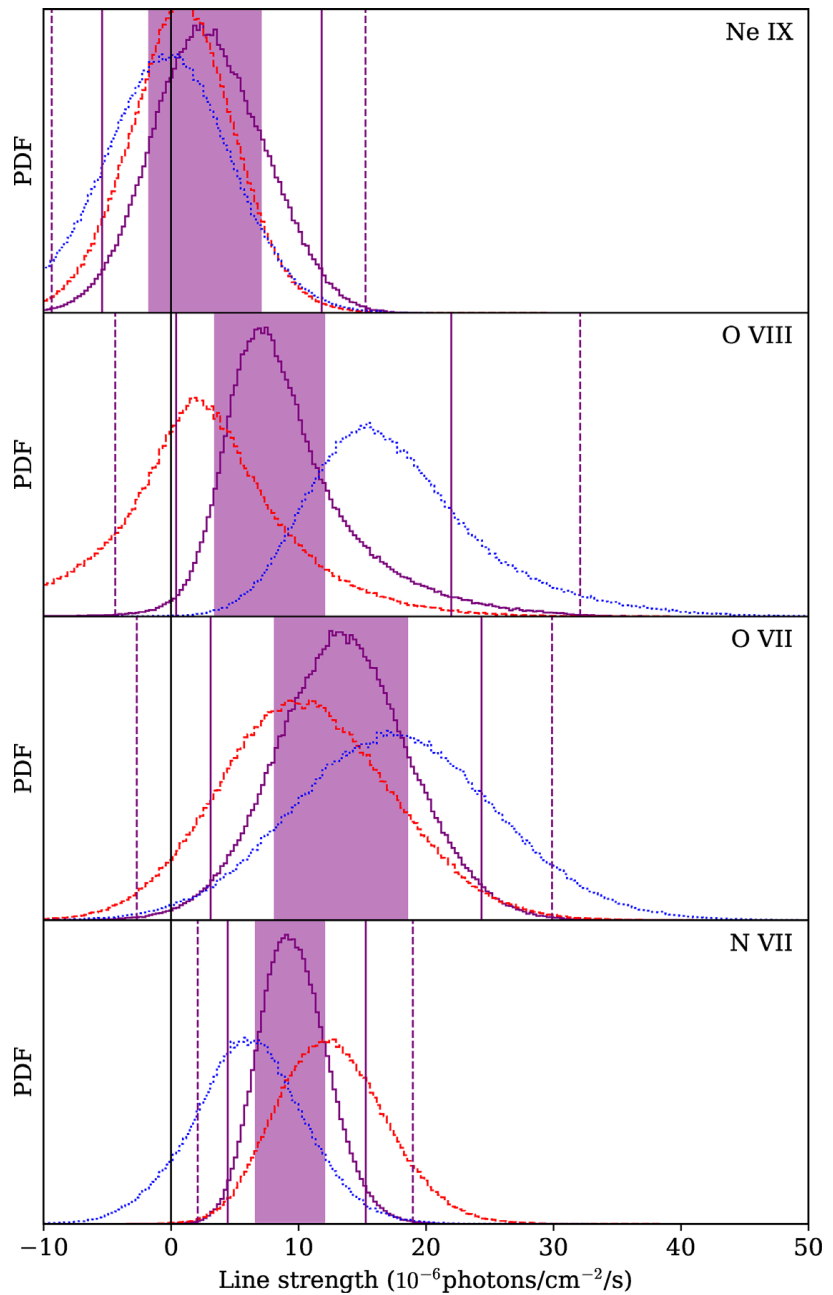

Figure 4. Posterior distributions of emission line strengths. For He-like triplets, the sum of all components is shown. Observation 2 is shown in red dashed lines, observation 7 in blue dotted lines, and the combined observation in purple lines. Shaded regions, solid, and dashed lines denote 68, 95, and 99.7 per cent Highest Posterior Density (HPD) credible regions, respectively.

range is $\left.n_{\mathrm{e}}=10^{10}-10^{12} \mathrm{~cm}^{-2}\right)$. Here, the intercombination line is the strongest, implying high-density photoionized plasma (since O VIII and N VII are detected with, at most, a small wavelength shift, it is unlikely that the apparent intercombination line is a blueshifted forbidden line).

We use the theoretical line ratios calculated by Porquet et al. (2001) to quantify this, comparing with the measured line ratio from the MCMC chains calculated in Section 3.1. Fig. 5 shows the constraint on density, from the ratio $R=z /(x+y)$. Since the forbidden line is not detected, the ratio $R$ is only constrained as an upper limit, which corresponds to a lower limit on the density. This shows that $n_{\mathrm{e}}>$ $4.1 \times 10^{10} \mathrm{~cm}^{-3}$ at 95 per cent credibility.

We also apply this method to the Ne IX line, but the line detections are not strong enough to provide a density constraint.

\subsection{Ionization from $\mathrm{O}$ VIII/O VII ratio}

The degree of ionization of the plasma is reflected in the relative strengths of lines from different ionization states of the same element. Here, we can use the relative flux in the O VII and O VIII lines.

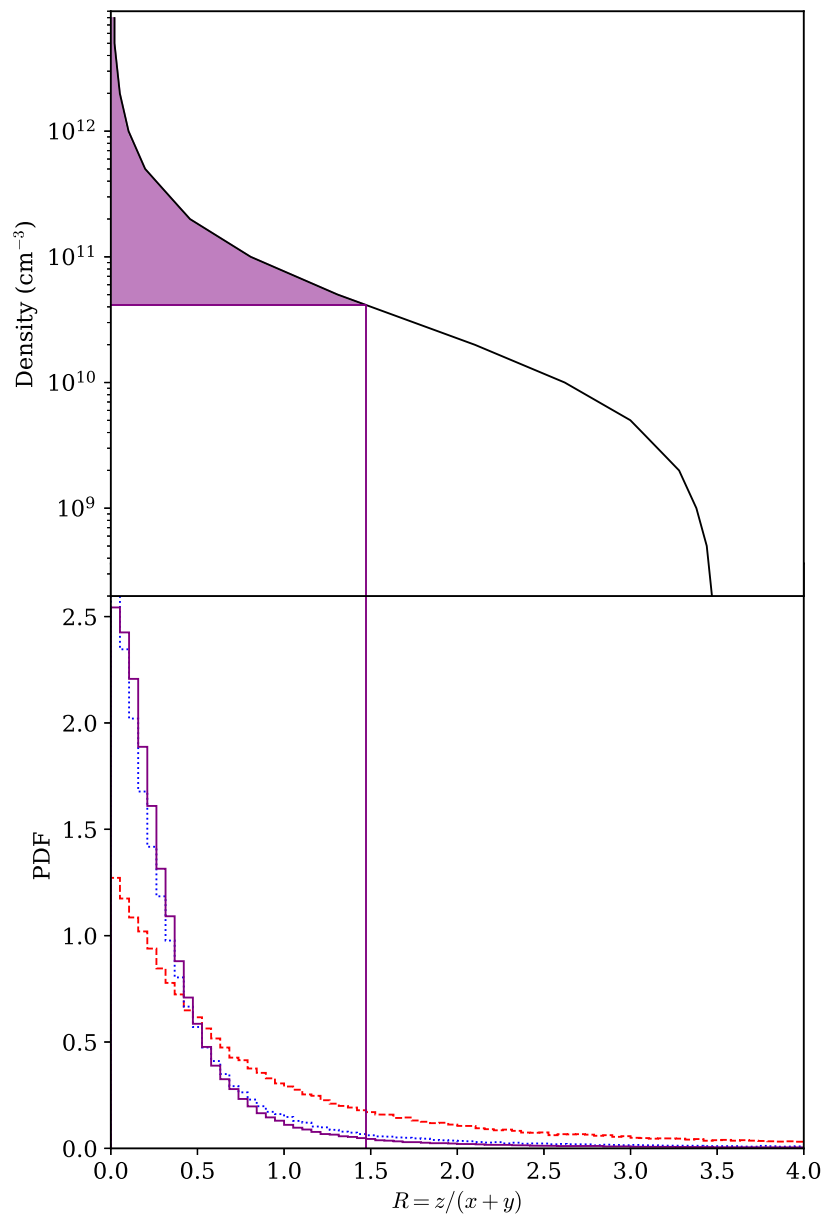

Figure 5. Constraint on density from the OVII triplet. Bottom panel: probability density of the forbidden $(z)$ to intercombination $(x+y)$ ratio; the vertical line marks the 95 percent upper limit. (observation 2 is shown in red dashed, observation 7 in blue dotted, and the combined observation in purple). Top panel: relation between line ratio and density, showing the corresponding lower limit to the density.

We calculate the relative strengths of the O VII and O VIII lines at different ionization parameters using the XSTAR package, version 2.5 (Kallman \& Bautista 2001). We calculate spectra over the range $\xi=$ $3-300 \mathrm{erg} \mathrm{cm} \mathrm{s}^{-1}$ in 20 logarithmically spaced steps and extract the flux for each line from the line luminosity file. We use an illuminating continuum based on fits to the NuSTAR spectrum (Hare et al. 2020) of observations in the same state as analysed here: a cut-off power law with $\Gamma=1.5$ and $E_{\text {Cut }}=30 \mathrm{keV}$. We use solar abundances and a density $n=10^{12} \mathrm{~cm}^{-3}$.

We then compare these predicted ratios with the ratio measured in the MCMC chains produced in Section 3.1. The distribution for each observation is shown in Fig. 6. This gives $\log (\xi)=$ $1.2_{-0.6}^{+0.5}$ for observation $201, \log (\xi)=1.4_{-0.2}^{+0.3}$ for observation 701 and $\log (\xi)=1.3_{-0.2}^{+0.3}$ (90 percent HPD) for the combined data set.

We also test the effects of density by repeating this process for $n=$ $10^{10} \mathrm{~cm}^{-3}$, but find that this has a very weak effect (Fig. 6). While the components of the O VII triplet change, their total flux remains similar in comparison to $\mathrm{O}$ VIII. 


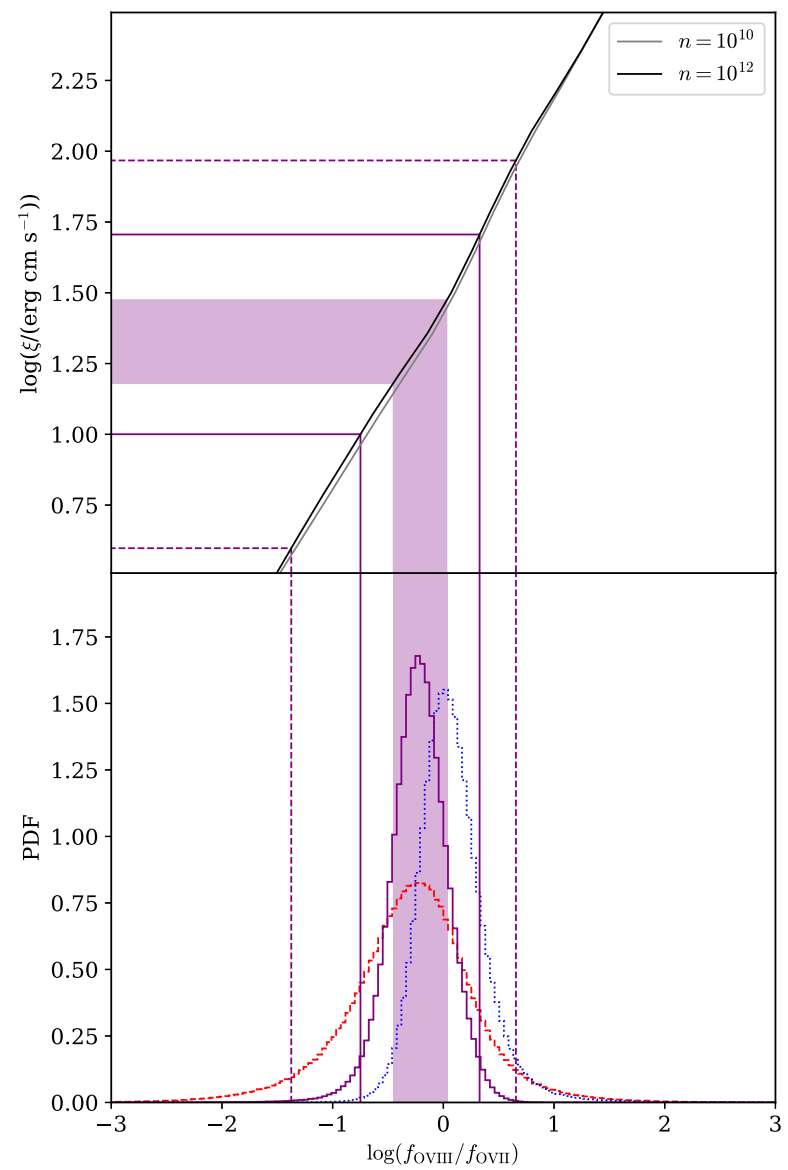

Figure 6. Constraint on the ionization parameter from the $\mathrm{O}$ VIII to $\mathrm{O}$ VII ratio. Bottom panel: probability density of the line flux ratio (observation 2 is shown in red dashed, observation 7 in blue dotted, and the combined observation in purple); the shaded regions and vertical lines mark $1 \sigma$ (68 per cent) and $2 \sigma$ (90 per cent) credible intervals, respectively for the combined observation. Top panel: relation between line ratio and ionization parameter, showing the corresponding constraints on the ionization. The black and grey lines show plasma densities of $n=10^{10}$ and $10^{12} \mathrm{~cm}^{-3}$, respectively; this has only a very small effect.

\subsection{Photoionized emission modelling}

As well as constraining the plasma properties from individual lines, we use a photoionization model to model the full spectrum. Using XSTAR, we construct a table model for the emission component with density and ionization as free parameters. We use 25 logarithmic steps over $n=10^{10}-10^{13} \mathrm{~cm}^{-3}$ and $\xi=3-1000 \mathrm{erg} \mathrm{cm} \mathrm{s}^{-1}$, with the same input continuum and ancillary parameters as in Section 3.4.
We fit a single instance of the emission component from this grid, along with a BLACKBODY+POWERLAW continuum model. However, the power law component is consistent with having zero flux (within 90 per cent errors), so we do not consider it further. The fit improvement of the model with emission over pure continuum is $\Delta C=25$. We also test the addition of a second photoionized emission component, but do not find a significant improvement $(\Delta C=1.7$ for 3 degrees of freedom).

Best-fitting parameters are given in Table 2 . The values of density, $\log \left(n / \mathrm{cm}^{3}\right)>11.2$, and ionization parameter, $\log \left(\xi /\right.$ erg $\left.\mathrm{cm} \mathrm{s}^{-1}\right)=$ $1.35 \pm 0.2$, for the combined data set are consistent with those measured from individual transitions.

We also perform an MCMC analysis based on this model. The posterior density for each parameter is shown in Fig. 7 and the median and HPD credible intervals in Table 2. This shows that there are no strong degeneracies between parameters and the derived values are similar to those found with traditional fitting methods.

\subsection{Derived properties}

From the quantities we have already measured, we may also place constraints on other important physical properties of the system. The distance from the source to the emitting plasma can be inferred by combining the luminosity, density, and ionization parameter

$R=\sqrt{L / n \xi}$

We have already measured the ionization and placed a lower limit on the density. The distance to Swift J1858.6-0814 has been estimated from the flux during photoshperic radius expansion of Type I X-ray bursts (Buisson et al. 2020b; Buisson et al., submitted) as around $15 \mathrm{kpc}$. This gives a luminosity $L \sim 2 \times 10^{38} \mathrm{erg} \mathrm{s}^{-1}$ (NuSTAR spectra taken at the times of the XMM-Newton spectra show a similar flux to that analysed in Hare et al. 2020). Combining this with the lower limits to density and ionization from observation 701 gives $R \lesssim 10^{13}(D / 15 \mathrm{kpc}) \mathrm{cm}$, corresponding to $5 \times 10^{7} \mathrm{r}_{\mathrm{g}}$ for a $1.4 \mathrm{M}_{\odot}$ neutron star.

A lower limit to the proximity of the emitting gas can be set by considering the amount of broadening observed in the line. Taking the line width as comparable to the Keplerian velocity gives $r / r_{\mathrm{g}} \sim(c / v)^{2}$; since the line may also be broadened by other effects, this estimate is a lower limit to the proximity of the emitting gas. For the most strongly constrained N VII line, $r>4 \times 10^{4} \mathrm{r}_{\mathrm{g}}=8 \times 10^{9}\left(M / 1.4 \mathrm{M}_{\odot}\right) \mathrm{cm}$.

\section{DISCUSSION}

We have found photoionized gas in Swift J1858.6-0814 with emission lines from Ne IX, O VII, O VIII, and N VII. From the properties of these lines, we can infer various physical parameters of the gas and its role in the accretion system of Swift J1858.6-0814; it is likely

Table 2. Parameters of fits to photoionization models. Normalizations are the XSPEC definitions; errors are given at 90 per cent confidence. We also show the values derived in previous sections from individual lines, which are consistent with the results from full photoionization modelling.

\begin{tabular}{lcccc}
\hline & $\begin{array}{c}\text { Observation } \\
\text { Data set }\end{array}$ & Observation & \\
& 201 & 701 & Combined & Combined \\
\hline Method & & Photoionization model fitting & From individual lines \\
$\log \left(n / \mathrm{cm}^{-3}\right)$ & $>10.6$ & $>10.5$ & $>11.3$ & $>10.6(95$ per cent $)$ \\
$\log \left(\xi / \mathrm{erg} \mathrm{cm} \mathrm{s}^{-1}\right)$ & $1.3_{-0.5}^{+1.2}$ & $1.5_{-0.4}^{+0.5}$ & $1.3_{-0.2}^{+0.4}$ & $1.3_{-0.2}^{+0.3}$ \\
Blackbody $(k T / \mathrm{keV})$ & $0.43_{-0.05}^{+0.06}$ & $0.47_{-0.06}^{+0.07}$ & $0.44_{-0.03}^{+0.05}$ & - \\
\hline
\end{tabular}




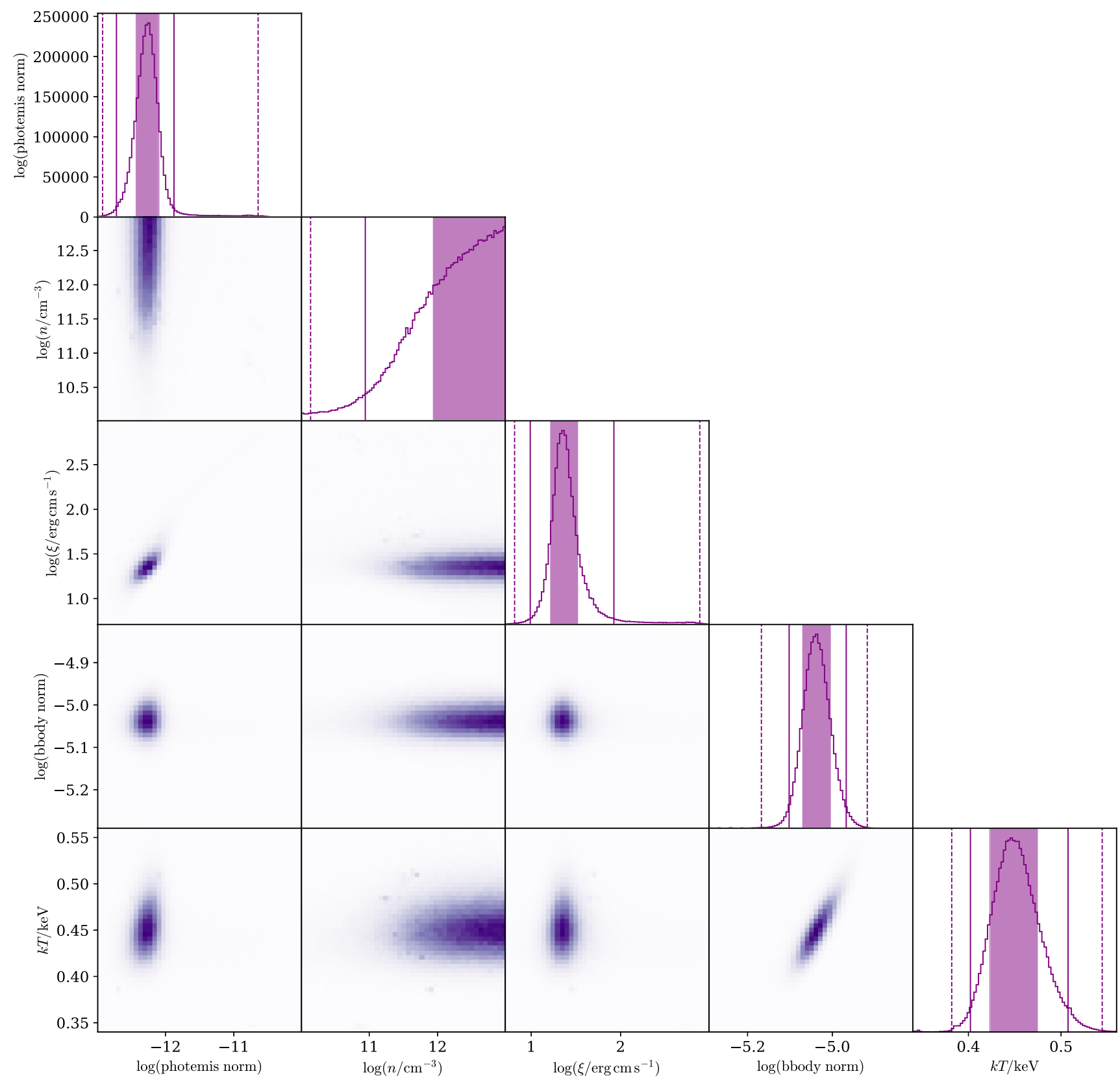

Figure 7. Posterior density plots from photoionization modelling. Normalizations are the XSPEC values. Shaded regions, solid, and dashed lines denote 68, 95 , and 99.7 per cent HPD credible regions, respectively.

to either be a hot accretion disc atmosphere or a component of the wind which is seen in other wavebands. The various constraints are discussed below.

We find that the density of the emitting plasma is $n_{\mathrm{e}} \geq$ $1.5 \times 10^{11} \mathrm{~cm}^{-3}$. While the effect of UV radiation on He-like triplet line ratios may bias our density measurement, high densities have also been found in X-ray absorbing winds from around black hole XRBs using Fe XXII line ratios (11.77 and $11.92 \AA$; see Mauche, Liedahl \& Fournier 2003), e.g. $\sim 10^{14} \mathrm{~cm}^{-3}$ (Miller et al. 2006a, 2008); $10^{16}-10^{17} \mathrm{~cm}^{-3}$ (Miller et al. 2014). Our limit is also consistent with other measurements of emitting plasma in X-ray binaries (Cottam et al. 2001; Kallman et al. 2003; Psaradaki et al. 2018) and is dense compared to many astrophysical objects. These constraints are, however, far from a typical binary disc value $\sim 10^{20}-10^{22} \mathrm{~cm}^{-3}$
(Svensson \& Zdziarski 1994; Tomsick et al. 2018; Jiang et al. 2019) so the emission is unlikely to be from the photosphere itself.

A similar emission component to that found here is detected by Psaradaki et al. (2018) in the neutron star LMXB EXO 0748-676: they find a similar ionization to that found here and use several Helike triplets to infer a plasma density of $>10^{13} \mathrm{~cm}^{-3}$. These lower ionization components (compared to many other XRBs), may be common but less studied due to Galactic absorption (Costantini et al. 2012; Díaz Trigo \& Boirin 2016). Psaradaki et al. (2018) also find a higher ionization $\left[\log \left(\xi / \mathrm{erg} \mathrm{cm} \mathrm{s}^{-1}\right) \sim 2.5\right]$ component, which could have an equivalent in Swift J1858.6-0814 responsible for the residuals matching higher ionization transitions that we find. A deeper high-resolution soft X-ray spectrum would be required to test this. 
The upper limit on the distance $\left(10^{13} \mathrm{~cm} \sim 5 \times 10^{7} \mathrm{r}_{\mathrm{g}}\right)$ of the emitting plasma from the neutron star is not highly constraining. For example, a typical launching radius seen in absorbing winds is $10^{3}-10^{4} \mathrm{r}_{\mathrm{g}}$ (e.g. Kallman et al. 2009). Similarly, where a disc wind is invoked as the material for the broad-line region (BLR) in a active galactic nucleus (AGN), it typically exists at around $10^{3}-10^{4} \mathrm{r}_{\mathrm{g}}$ (Tremaine et al. 2014). However, these sizes are typically for absorbing material detected with higher ionization, which is likely to occur closer to the central source: The emission observed here could very well be located further from it.

We also have a lower limit on the size of the emitting region from the narrowness of the lines: This implies that the majority of the N VII emission comes from $r>4 \times 10^{4} \mathrm{r}_{\mathrm{g}}$. It is possible that the lines are emitted closer to the neutron star with broader profiles but their blue wing is absorbed. However, absorbing the whole of the blue side would only allow for a change by a factor of $\sim 2$ in velocity and $\sim 4$ in radius. This distance constraint is at the upper end of AGN BLR radii, so the component we observe may be closer to an analogue of an AGN narrow-line region (NLR; in terms of scale in $r_{\mathrm{g}}$; AGN NLR densities are much lower, typically $<10^{5} \mathrm{~cm}^{-3}$, Bennert et al. 2006).

As well as the size, we can also consider the shape of the emitting material. Disc winds in binaries are predicted to include material ejected in all directions (e.g. Higginbottom et al. 2018); the denser regions producing X-ray signatures are believed to be predominantly equatorial, since they are usually seen in absorption in high inclination sources (Boirin et al. 2005; Miller et al. 2006a,b; King et al. 2012; Ponti et al. 2012). Swift J1858.6-0814 shows periodic eclipses, which shows that it is at high inclination $\left(>70^{\circ}\right.$, Buisson et al. 2020b, Buisson et al., in preparation). This can explain the emission line properties (c.f. Charles et al. 2019): If much of the X-ray continuum is blocked by the disc, even a modestly high scattering atmosphere will produce observable emission lines. In this situation, the unusually strong variability of Swift J1858.6-0814 can be explained as variable obscuration from surface of a rough disc viewed at very high inclination: as the material in the disc orbits, thicker obscuring regions move into and out of the line of sight. Also, the surface of the disc will include more dense material, which can maintain sufficiently low ionization to produce optical absorption features. This scenario may be able to explain the observed optical wind (Muñoz-Darias et al. 2020).

We also find some evidence for the emission lines being redshifted $[P(z>0)=0.96]$, largely due to the N VII line $[P(z>0)=0.96]$. This may include contributions from the absolute calibration of the RGS and the radial velocity of the source. The RGS is calibrated to $6 \mathrm{m \AA},{ }^{1}$ corresponding to $\pm 80 \mathrm{~km} \mathrm{~s}^{-1}$ at the wavelength of $\mathrm{N}$ VII. There is no evidence for significant radial velocity in the optical spectra (Muñoz-Darias et al. 2020) and most X-ray binaries have a radial velocity of $\lesssim 100 \mathrm{~km} \mathrm{~s}^{-1}$ (Gandhi et al. 2019). Allowing a conservative $200 \mathrm{~km} \mathrm{~s}^{-1}$ for these effects still leaves a likely redshift, $P\left(z>200 \mathrm{~km} \mathrm{~s}^{-1}\right)=0.89$. If there is an intrinsic source redshift, it could be due to the blue wing of the line being absorbed to form a P-Cygni profile as is also seen in the optical spectra (Muñoz-Darias et al. 2020). If this is the case, the redward extent of the line can be used to estimate the wind velocity: combining the width of the line with its redshift gives $v \sim 2000 \mathrm{~km} \mathrm{~s}^{-1}$, similar to other wind velocities. An alternative interpretation is that the line is emitted by gravitationally redshifted or infalling material: Miller et al. (2014) find (although in a compromised observation) redshifted absorption

${ }^{1}$ https://xmm-tools.cosmos.esa.int/external/xmm_user_support/documentati on/uhb/rgswavscal.html components in MAXI J1305-704 corresponding to the free-fall and gravitational redshift of material at around $500 \mathrm{r}_{\mathrm{g}}$. Since the lines observed here are seen in emission, an inflow is less likely since there would need to be a large central structure blocking the blueshifted emission from the far side. Alternatively, the redshifted emission could occur where the accretion stream impacts the disc, as suggested by Psaradaki et al. (2018).

To directly confirm the X-ray P-Cygni profile by observing the absorption would require unfeasibly long RGS observations, but could be possible with brighter sources or future telescopes with larger effective areas.

\section{ACKNOWLEDGEMENTS}

We thank the referee for useful comments which have improved the clarity of the manuscript. DA and DJKB acknowledge support from the Royal Society. MAP is funded by the Juan de la Cierva (Spanish State Research Agency) Fellowship IJCI-2016-30867. TMD is funded by the Ramón y Cajal Fellowship RYC-2015-18148. MAP and TMD acknowledge support by the Spanish MINECO grant AYA2017-83216-P. NCS and CK acknowledge support by the Science and Technology Facilities Council (STFC) and from STFC grant ST/M001326/1. JvdE and ND are supported by a Vidi grant from the Netherlands Organisation for Scientific research (NWO), awarded to ND. FF and DA acknowledge support from the Royal Society International Exchanges 'The first step for HighEnergy Astrophysics relations between Argentina and UK'. This work has made use of observations obtained with XMM-Newton, an ESA science mission with instruments and contributions directly funded by ESA Member States and NASA. This research has made use of ISIS functions (ISISscripts) provided by ECAP/Remeis observatory and MIT (http://www.sternwarte.uni-erlangen.de/isis/). MOA acknowledges support from the Royal Society through the Newton International Fellowship programme. FMV acknowledges support from STFC under grant ST/R000638/1.

\section{DATA AVAILABILITY}

The data on which this work is based are available from the $X M M-N e w t o n$ Science Archive.

\section{REFERENCES}

Begelman M. C., McKee C. F., Shields G. A., 1983, ApJ, 271, 70

Bennert N., Jungwiert B., Komossa S., Haas M., Chini R., 2006, A\&A, 459, 55

Blandford R. D., Payne D. G., 1982, MNRAS, 199, 883

Boirin L., Méndez M., Díaz Trigo M., Parmar A. N., Kaastra J. S., 2005, A\&A, 436, 195

Bright J., Fender R., Motta S., Rhodes L., Titterington D., Perrott Y., 2018, Astron. Telegram, 12184, 1

Buisson D. J. K., Altamirano D., Remillard R., Arzoumanian Z., Gendreau K., Gandhi P., Vincentelli F., 2020a, Astron. Telegram, 13536, 1

Buisson D. J. K. et al., 2020b, Astron. Telegram, 13563, 1

Casares J., Muñoz-Darias T., Mata Sánchez D., Charles P. A., Torres M. A. P., Armas Padilla M., Fender R. P., García-Rojas J., 2019, MNRAS, 488, 1356

Cash W., 1979, ApJ, 228, 939

Charles P., Matthews J. H., Buckley D. A. H., Gandhi P., Kotze E., Paice J., 2019, MNRAS, 489, L47

Costantini E. et al., 2012, A\&A, 539, A32

Cottam J., Sako M., Kahn S. M., Paerels F., Liedahl D. A., 2001, ApJ, 557, L101 
Díaz Trigo M., Boirin L., 2013, Acta Polytech., 53, 659

Díaz Trigo M., Boirin L., 2016, Astron. Nachr., 337, 368

Dorodnitsyn A. V., 2009, MNRAS, 393, 1433

Dorodnitsyn A. V., 2010, MNRAS, 406, 1060

Fender R., Muñoz-Darias T., 2016, The Balance of Power: Accretion and Feedback in Stellar Mass Black Holes, Springer-Verlag, Berlin, p. 65

Foreman-Mackey D., Hogg D. W., Lang D., Goodman J., 2013, PASP, 125, 306

Gandhi P. et al., 2016, MNRAS, 459, 554

Gandhi P., Rao A., Johnson M. A. C., Paice J. A., Maccarone T. J., 2019, MNRAS, 485, 2642

Goodman J., Weare J., 2010, Commun. Appl. Math. Comput. Sci., 5, 65

Hare J., Gandhi P., Paice J. A., Tomsick J., 2019, Astron. Telegram, 12512, 1

Hare J. et al., 2020, ApJ, 890, 16

Higginbottom N., Knigge C., Long K. S., Matthews J. H., Sim S. A., Hewitt H. A., 2018, MNRAS, 479, 3651

Houck J. C., Denicola L. A., 2000, in Manset N., Veillet C., Crabtree D., eds, Conf. Ser. Vol. 216, Astronomical Data Analysis Software and Systems IX. Astron. Soc. Pac., San Francisco, p. 591

Icke V., 1980, AJ, 85, 329

Jansen F. et al., 2001, A\&A, 365, L1

Jiang J., Fabian A. C., Wang J., Walton D. J., García J. A., Parker M. L., Steiner J. F., Tomsick J. A., 2019, MNRAS, 484, 1972

Jimenez-Garate M. A., Raymond J. C., Liedahl D. A., 2002, ApJ, 581, 1297

Kafka S., Honeycutt R. K., 2004, AJ, 128, 2420

Kallman T., Bautista M., 2001, ApJS, 133, 221

Kallman T. R., Angelini L., Boroson B., Cottam J., 2003, ApJ, 583, 861

Kallman T. R., Bautista M. A., Goriely S., Mendoza C., Miller J. M., Palmeri P., Quinet P., Raymond J., 2009, ApJ, 701, 865

King A. L. et al., 2012, ApJ, 746, L20

King A. L., Miller J. M., Raymond J., Reynolds M. T., Morningstar W., 2015, ApJ, 813, L37

Krimm H. A. et al., 2018, Astron. Telegram, 12151, 1

Mauche C. W., Liedahl D. A., Fournier K. B., 2003, ApJ, 588, L101

Miller J. M., Raymond J., Fabian A., Steeghs D., Homan J., Reynolds C., van der Klis M., Wijnands R., 2006a, Nature, 441, 953

Miller J. M. et al., 2006b, ApJ, 646, 394

Miller J. M., Raymond J., Reynolds C. S., Fabian A. C., Kallman T. R., Homan J., 2008, ApJ, 680, 1359

Miller J. M. et al., 2014, ApJ, 788, 53

Miller J. M., Fabian A. C., Kaastra J., Kallman T., King A. L., Proga D., Raymond J., Reynolds C. S., 2015, ApJ, 814, 87

Miller J. M. et al., 2016, ApJ, 821, L9

Miller K. A., Stone J. M., 2000, ApJ, 534, 398
Motta S. E., Kajava J. J. E., Sánchez-Fernández C., Giustini M., Kuulkers E., 2017, MNRAS, 468, 981

Muñoz-Darias T. et al., 2016, Nature, 534, 75

Muñoz-Darias T. et al., 2020, ApJ, 893, 10

Muñoz-Darias T., Torres M. A. P., Garcia M. R., 2018, MNRAS, 479, 3987

Munoz-Darias T., Jimenez-Ibarra F., Armas Padilla M., Casares J., Cuneo V., Panizo-Espinar G., Sanchez-Sierras J., Torres M. A. P., 2019, Astron. Telegram, 12881, 1

Neilsen J., Remillard R. A., Lee J. C., 2011, ApJ, 737, 69

Paice J. A., Gandhi P., Dhillon V. S., Marsh T. R., Green M., Breedt E., 2018, Astron. Telegram, 12197, 1

Ponti G., Fender R. P., Begelman M. C., Dunn R. J. H., Neilsen J., Coriat M., 2012, MNRAS, 422, L11

Porquet D., Mewe R., Dubau J., Raassen A. J. J., Kaastra J. S., 2001, A\&A, 376,1113

Prinja R. K., Ringwald F. A., Wade R. A., Knigge C., 2000, MNRAS, 312, 316

Proga D., Kallman T. R., 2002, ApJ, 565, 455

Protassov R., van Dyk D. A., Connors A., Kashyap V. L., Siemiginowska A., 2002, ApJ, 571, 545

Psaradaki I., Costantini E., Mehdipour M., Díaz Trigo M., 2018, A\&A, 620, A129

Puebla R. E., Diaz M. P., Hillier D. J., Hubeny I., 2011, ApJ, 736, 17

Rajwade K. M. et al., 2019, Astron. Telegram, 12499, 1

Revnivtsev M., Gilfanov M., Churazov E., Sunyaev R., 2002, A\&A, 391, 1013

Reynolds M. T., Miller J. M., Ludlam R. M., Tetarenko B. E., 2018, Astron. Telegram, 12220, 1

Schulz N. S., Brandt W. N., 2002, ApJ, 572, 971

Svensson R., Zdziarski A. A., 1994, ApJ, 436, 599

Tomsick J. A. et al., 2018, ApJ, 855, 3

Tremaine S., Shen Y., Liu X., Loeb A., 2014, ApJ, 794, 49

Ueda Y., Inoue H., Tanaka Y., Ebisawa K., Nagase F., Kotani T., Gehrels N., 1998, ApJ, 492, 782

van den Eijnden J. et al., 2020, MNRAS, 496, 4127

Vasilopoulos G., Bailyn C., Milburn J., 2018, Astron. Telegram, 12164, 1

Walton D. J. et al., 2017, ApJ, 839, 110

White N. E., Holt S. S., 1982, ApJ, 257, 318

Wijnands R., van der Klis M., 2000, ApJ, 528, L93

Woods D. T., Klein R. I., Castor J. I., McKee C. F., Bell J. B., 1996, ApJ, 461,767

This paper has been typeset from a $\mathrm{T}_{\mathrm{E}} \mathrm{X} / \mathrm{LT} \mathrm{T}_{\mathrm{E}} \mathrm{X}$ file prepared by the author. 